\title{
¿Qué actitudes hacia la ciencia posee el alumnado de Educación Primaria que participa en actividades científicas extracurriculares?
}

\author{
Radu Bogdan Toma, Jairo Ortiz-Revilla e lleana M. Greca \\ Departamento de Didácticas Específicas, Facultad de Educación, Universidad de Burgos, \\ Burgos, España
}

[Recibido el 19 de octubre de 2018, aceptado el 27 de diciembre de 2018]

La necesidad de impulsar la alfabetización científica desde edades tempranas y el generalizado descenso del interés por seguir carreras científico-tecnológicas representan algunas de las principales problemáticas de la investigación en Didáctica de las Ciencias. En este estudio se evalúan las actitudes hacia la ciencia de 960 estudiantes de 3 a 6o curso de Educación Primaria que asisten a actividades extracurriculares de ciencias. Los resultados muestran que, en general, estos estudiantes poseen actitudes favorables hacia la ciencia, sin embargo, su entusiasmo por la ciencia y sus actitudes hacia las clases de Ciencias de la Naturaleza descienden con el aumento del nivel escolar. La diferencia entre las actitudes de niños y niñas no es tan marcada como en la literatura existente, identificándose inclusive que las niñas poseen actitudes más positivas hacia los científicos. Estos resultados subrayan la necesidad de tomar medidas educativas enfocadas a prevenir el descenso de las actitudes de los estudiantes desde los cursos elementales del sistema educativo.

Palabras clave: actitudes hacia la ciencia; actitudes hacia la ciencia escolar; Educación Primaria; actividades científicas extracurriculares.

\section{What are the attitudes toward science of elementary students participating in extracurricular science activities?}

The need to promote scientific literacy since childhood and the general decline in interest in pursuing scientific-technological careers represent some of the main problems of research in science education. In this study, the attitudes towards science of 960 students enrolled in 3rd to 6th grades of Elementary Education who attend extracurricular science activities are evaluated. The results show that, in general, these students have favorable attitudes towards science, however, their enthusiasm in science and their attitudes towards science classes decrease with the increase of the school level. The difference in attitudes of boys and girls is not as marked as in existing literature studies, even identifying that girls have more positive attitudes towards scientists. These results highlight the need to take educational measures to prevent the decline of students' attitudes from the elementary courses.

Keywords: attitudes towards science; attitudes towards school science; Elementary Education; extracurricular scientific activities.

Para citar el artículo. Toma, R. B., Ortiz-Revilla, J., y Greca, I. M. (201x). ¿¿Qué actitudes hacia la ciencia posee el alumnado de Educación Primaria que participa en actividades científicas extracurriculares? Ápice. Revista de Educación Científica, 3(1), 55-69. DOI: https://doi.org/10.17979/ arec.2019.3.1.4599

Contacto.rbtoma@ubu.es, jortizr@ubu.es, imgreca@ubu.es 


\section{Introducción}

La comunidad científica conoce con certeza que desde hace algunos años se ha venido registrando un desinterés generalizado de los estudiantes por cuestiones científico-tecnológicas, situación preocupante ya que presenta repercusiones directas en el desarrollo adecuado de la alfabetización científica de las personas (Osborne y Dillon, 2008) y está provocando un descenso de las matrículas registradas en las carreras universitarias de esta índole (EC, 2015). Las investigaciones dedicadas a abordar la problemática del descenso de las actitudes positivas hacia la ciencia parecen mostrar que el período de 10 a 14 años - coincidente con los últimos cursos de la etapa de Educación Primaria - resulta crítico en el moldeado de las actitudes y las aspiraciones de los estudiantes hacia carreras científicas (Tai, Liu, Maltés y Fan, 2006). No obstante, en España existen pocos estudios enfocados a estudiar las actitudes hacia la ciencia de los estudiantes de esta etapa educativa, sobre todo desde las edades tempranas. La mayoría de los estudios están principalmente dirigidos a la etapa de Educación Secundaria Obligatoria y en adelante, existiendo algunos que no incluyen muestra de alumnado de Educación primaria (Pérez-Franco, de Pro-Bueno y Pérez-Manzano, 2018; Vázquez Alonso, Acevedo Díaz, Manassero Mas y Acevedo Romero, 2006) y otros cuyo límite muestral inferior corresponde al sexto curso de la Educación Primaria (Marbà-Tallada y Márquez Bargalló, 2010; Nortes MartínezArtero y de Pro Bueno, 2010; Pérez Manzano y de Pro Bueno, 2018; de Pro Bueno y Pérez Manzano, 2014) o en el caso de Vázquez Alonso y Manassero Mas (1997) con estudiantes de 13-14 años. Únicamente, Vázquez Alonso y Manassero Mas (2008) analizaron las actitudes hacia la ciencia de una muestra que englobaba a 221 alumnos desde el cuarto curso de la Educación Primaria.

Dada la necesidad de ahondar en este sentido, el objetivo principal de esta investigación es diagnosticar las actitudes hacia la ciencia que posee el alumnado de 30 a 60 curso de Educación Primaria en el contexto español. La muestra está conformada por estudiantes que participan voluntariamente en actividades extracurriculares de ciencia y al tratarse de una amplia muestra con características poco investigadas, el presente trabajo puede aportar luz al estudio de este constructo y así, poder velar por la mejora de la alfabetización científica temprana del alumnado sin que su desarrollo competencial quede relegado a etapas escolares posteriores (Ortiz-Revilla, Greca y Adúriz-Bravo, 2017, 2018). Las preguntas de investigación planteadas en este estudio son:

- P1. ¿Cuál es la actitud hacia la ciencia de los estudiantes de Educación Primaria interesados en actividades extracurriculares?

- P2. ¿Difieren las actitudes hacia la ciencia según el género de los estudiantes?

- P3. ¿Difieren las actitudes hacia la ciencia según el nivel escolar?

\section{Antecedentes teóricos}

\section{El constructo "actitudes hacia la ciencia"}

El estudio del panorama de la investigación acerca de las actitudes hacia la ciencia constituye una dificultad en sí mismo, ya que su abordaje no consiste en explorar una única dimensión dado que se considera que varios subconstructos o dimensiones contribuyen en mayor o menor medida al desarrollo de las actitudes de un determinado individuo hacia la ciencia. Por ello, dependiendo del enfoque teórico adoptado, numerosos autores han abordado diversas dimensiones que podrían influir en el desarrollo de sus actitudes. Las dimensiones más estudiadas en cuanto a su influencia en las actitudes hacia la ciencia están relacionadas con la implicación social de la ciencia, el disfrute de la ciencia, el interés por cuestiones científicas y el deseo por continuar carreras científicas 
(Akpınar, Yıldız, Tatar y Ergin, 2009; Ali, Yager, Hacieminoglu y Caliskan, 2013; Caleon y Subramaniam, 2005, 2008; Denessen, Vos, Hasselman, y Louws, 2015; Guzey, Moore, Harwell y Moreno, 2016; Huang, Chiu y Hong, 2016; Jarvis y Pell, 2005; Mıhladız, Duran y Doğan, 2011; Molina, Carriazo y Casas, 2013; Pérez Manzano y de Pro Bueno, 2018; Vázquez Alonso et al., 2006). Otros autores han incluido dimensiones relacionadas de forma directa con la ciencia escolar y cómo la percepción de autoeficacia del alumnado influye en sus actitudes hacia la ciencia (DeWitt y Archer, 2015; George, 2006; Hacieminoglu, 2016; Jones, Howe y Rua, 2000; Marbà-Tallada y Márquez Bargalló, 2010; Newell, Zientek, Tharp, Vogt y Moreno, 2015; Vázquez Alonso y Manassero Mas, 2008). Una minoría de estudios han estudiado otras dimensiones como la credibilidad y la repercusión social de las ciencias (de Pro Bueno y Pérez Manzano, 2014), las actitudes ambientales (Pérez-Franco et al., 2018), la presión de los profesores y los padres para el éxito en las materias de ciencias (George, 2006), o la influencia de los roles adoptados entre iguales (Newell et al., 2015).

\section{Actitudes hacia la ciencia en educación primaria}

Las investigaciones realizadas sobre las actitudes hacia la ciencia en estudiantes de Educación Primaria apuntan a una multiplicidad de variables influyentes en su desarrollo desde edades tempranas, entre las que destacan el género y la edad.

En cuanto al género, la mayoría de los estudios existentes en la literatura han mostrado que los niños presentan mejores actitudes hacia la ciencia que las niñas (Boone, 1997; Caleon y Subramaniam, 2008; Denessen et al., 2015; DeWitt y Archer, 2015; George, 2006; Hacieminoglu, 2016; Jarvis y Pell, 2005; Pérez Manzano y de Pro Bueno, 2018; de Pro Bueno y Pérez Manzano, 2014; Vázquez Alonso y Manassero Mas, 2008). Por ejemplo, de Pro Bueno y Pérez Manzano (2014), en un estudio nacional realizado con una muestra de 6827 estudiantes, mostraron que los niños presentaban posiciones más positivas que las niñas respecto a la valoración de noticias científicas y la creencia hacia una mejora del estilo de vida promovido por la ciencia. Las niñas suelen presentar niveles más bajos de disfrute en el aprendizaje de la ciencia y la tecnología (Denessen et al., 2015), de disfrute de las clases de ciencias (Marbà-Tallada y Márquez Bargalló, 2010) y se manifiestan más indecisas sobre su preferencia por las carreras relacionadas con estas (Caleon y Subramaniam, 2008). Sin embargo, los niños muestran aspiraciones científicas más sólidas (DeWitt y Archer, 2015). En contraste con estos resultados, un estudio realizado por Akpınar et al. (2009) encontró más interés hacia la ciencia entre las participantes niñas; no obstante, no fue así en cuanto a los factores de ansiedad y disfrute de los experimentos científicos. Mıhladız et al. (2011) encontraron diferencias significativas a favor de las niñas de 11 años, que mostraron mejores actitudes en cuanto al interés y la curiosidad por cuestiones científicas y por el disfrute de actividades experimentales. Recientemente, Pérez-Franco et al. (2018) han registrado actitudes más favorables en las chicas en la mayoría de las cuestiones relacionadas con las actitudes ambientales, reflejando más responsabilidad, concienciación y respeto, aunque en los chicos estas resultan también positivas. A pesar de que, por lo general, los niños presentan mejores actitudes hacia la ciencia, George (2006) encontró que sus actitudes decrecen más rápidamente que las de las niñas a medida que aumenta el nivel escolar. Como apunte, pocos estudios informan de una ausencia de efecto del género sobre las actitudes de los estudiantes de Educación Primaria hacia la ciencia (Khishfe y Boujaoude, 2016; Nortes Martínez-Artero y de Pro Bueno, 2010; Said, Summers, Abd-El-Khalick y Wang, 2016). Babaoğlan y Arıkan (2017) tampoco han encontrado diferencias significativas entre las actitudes hacia la ciencia escolar de los niños y las niñas. No obstante, Khisfe y Boujaoude (2016) han encontrado diferencias significativas de género en cuanto a las concepciones científicas. 
En relación con la edad y el nivel académico, la literatura reporta datos consistentes acerca de un patrón decreciente en las actitudes positivas de los estudiantes hacia la ciencia a medida que aumenta el curso escolar (Akpınar et al., 2009; Ali et al., 2013; Denessen et al., 2015; DeWitt y Archer, 2015; George, 2006;Marbà-Tallada y Márquez Bargalló, 2010;Mıhladız et al., 2011; Molina et al., 2013; de Pro Bueno y Pérez Manzano, 2014;Said et al., 2016; Vázquez Alonso y Manassero Mas, 2008). Aunque las actitudes hacia la ciencia escolar disminuyen drásticamente en la etapa de Educación Secundaria, las actitudes hacia la importancia de la ciencia parecen seguir siendo positivas (Akpınar et al., 2009; George, 2006), cuestión que resulta alentadora. Sin embargo, los estudiantes de Educación Primaria de cursos superiores y de los dos primeros años de Secundaria suelen mostrar actitudes negativas hacia el disfrute de las clases de ciencias (Ali et al., 2013; DeWitt y Archer, 2015), especialmente durante el segundo nivel, momento en el que se produce la mayor disminución de las actitudes hacia la ciencia (Akpınar et al., 2009) y hacia las clases de ciencias (Marbà-Tallada y Márquez Bargalló, 2010). Por ejemplo, Caleon y Subramaniam (2008) registraron un alto impacto del rendimiento académico en las actitudes hacia la ciencia de estudiantes con edades entre 10 y 12 años, cuestión que podría servir como explicación de la aparición de las actitudes negativas a estas edades. Destacar que aquellos estudiantes con calificaciones bajas y medias mostraron peores actitudes en comparación con aquellos que obtuvieron buenas calificaciones y las actitudes de estos eran muy parecidas a los estudiantes con altas capacidades.

También existen investigaciones con diferentes enfoques. DeWitt y Archer (2015) estudiaron las aspiraciones de 13.919 estudiantes de 11 a 14 años por carreras científicas, concluyendo que las actitudes positivas de los niños hacia la ciencia escolar y las actitudes positivas de los padres hacia la ciencia no se traducían en un mayor deseo por seguir estudios científicos. Por otro lado, Denessen et al. (2015) se centraron en estudiar los efectos de las actitudes de los profesores hacia la enseñanza de las ciencias sobre las actitudes de los estudiantes hacia el disfrute de las clases de ciencia y su autopercepción de eficacia. Los resultados indicaron que parecía existir una relación directa entre los estudiantes que desarrollaban peores actitudes hacia la ciencia escolar durante la etapa de Educación Primaria -especialmente en el caso de las niñas- y los maestros que poseían un bajo interés por la ciencia.

\section{Método}

\section{Muestra y contexto}

Un total de 1005 alumnos de Educación Primaria participaron en este estudio. Los participantes acudieron a una actividad extracurricular no obligatoria llamada Sábados de Ciencia. Esta actividad consiste en realizar un taller de ciencias durante tres horas, un sábado del mes (Greca, Diez-Ojeda y García Terceño, en revisión). Los estudiantes provienen de 19 centros -11 públicos y 6 concertados de la capital y dos públicos del interior de la provincia de Burgos-. Se trata de una muestra por conveniencia, dado que tanto los centros como los estudiantes escogen participar voluntariamente en la actividad. Atendiendo al número total de centros existentes en la provincia de Burgos (JCyL, 2018), cabe destacar que el número de centros participantes es representativo de la oferta educativa para la enseñanza primaria de la provincia de Burgos pues corresponden al $16 \%$ de los centros de enseñanza primaria de toda la provincia y al $42,5 \%$ de los centros de la capital. Tras descartar casos no válidos por respuestas vacías o más de una respuesta por ítem, el total de cuestionarios válidos fue de 960. La Tabla 1 recoge la distribución de los participantes según el género y el curso escolar. 
Tabla 1. Muestra según género y curso escolar

\begin{tabular}{|l|c|c|c|c|c|}
\hline \multirow{2}{*}{ Género } & \multicolumn{4}{|c|}{ Cursos de Educación Primaria } & \multirow{2}{*}{ Total } \\
\cline { 2 - 5 } & 3o & 4 ㅇ & 5 & 6 \% & \\
\hline Niña & 97 & 154 & 144 & 128 & 523 \\
\hline Niño & 103 & 115 & 131 & 86 & 437 \\
\hline Total & 202 & 269 & 275 & 214 & 960 \\
\hline
\end{tabular}

\section{Instrumento}

Se empleó el cuestionario utilizado por Toma y Greca (2018) y Toma, Greca y Orozco Gómez (2019). Se trata de una escala de 14 ítems adaptada a partir del instrumento TOSRA de Fraser (1981). EI TOSRA emplea la clasificación de las dimensiones de actitudes hacia la ciencia propuestas por Klopfer (1971) y pretende examinar las actitudes de los estudiantes a través de 70 ítems de tipo Likert correspondientes a siete factores: (i) implicación social de la ciencia, (ii) normalidad de los científicos, (iii) actitudes hacia la investigación científica, (iv) adopción de actitudes científicas, (v) disfrute de las clases de ciencias, (vi) interés por un ocio en ciencias, y (vii) actitudes hacia una carrera en ciencias. Toma y Greca (2018) adaptaron la escala al contexto de una intervención educativa desarrollada con estudiantes de 4을 curso de Educación Primaria españoles. Para que el cuestionario fuera más viable para los estudiantes de niveles elementales, los autores redujeron la escala a un total de 14 ítems, dos por cada factor. En primer lugar, eliminaron los ítems formulados negativamente. A continuación, seleccionaron cuatro ítems por constructo mediante consenso. Por último, realizaron un estudio piloto con 24 estudiantes de 4을 curso de Educación Primaria, tras el cual eliminaron los ítems con baja consistencia interna $(\alpha<.60)$, conformándose la escala final de 14 ítems, con un $\alpha$ de Cronbach de .78 .

\section{Análisis de datos}

Toma y Greca (2018) emplearon una muestra de 96 estudiantes y Toma, Greca y OrozcoGómez una muestra de 118 participantes, siendo ambas muy escasas y, por tanto, no aptas para realizar análisis factorial. Por ello, en este estudio, los 14 ítems del cuestionario adaptado TOSRA fueron sometidos a un análisis de componentes principales (ACP). Dado que en el cuestionario original algunos factores estaban altamente correlacionados, se empleó una rotación oblicua Promax, que permite alcanzar soluciones con estructuras lo más cercanas a la ideal (Lloret-Segura, Ferreres-Traves, Hernández-Baeza y Tomás-Marco, 2014; Méndez Martínez y Rondón Sepúlveda, 2012). Se empleó la prueba de esfericidad de Bartlett y la prueba Kaiser-Meyer-Olkin (KMO) para determinar la idoneidad de la muestra y de los datos para la extracción factorial, con un criterio KMO > .60 y $\mathrm{p}<.001$ considerado como aceptable (Tabachnick y Fidell, 2007). El número de factores a retener se determinó mediante el criterio Kaiser > 1 (Kaiser, 1970). Se empleó un valor mínimo de .40 para la inclusión de cada ítem en su correspondiente factor.

Las respuestas de los participantes se analizaron mediante estadística descriptiva, la prueba $t$ de Student para muestras independientes - variable género- y la prueba de ANOVA - variable curso escolar-. Se aplicó la corrección de Bonferroni para ajustar el valor de $p$ y reducir la probabilidad de cometer errores de tipo I. Así, se estableció el nivel de significancia estadística en $p=.01$. 


\section{Resultados}

\section{Validación del instrumento}

El valor KMO fue de .803 y la prueba de esfericidad de Bartlett alcanzó significancia estadística $(p<.001)$, apoyando la idoneidad de la muestra y de los datos para la extracción factorial. El ACP reveló la existencia de cuatro factores con valores propios $K>1$, aunque algunos ítems resultaron problemáticos. El ítem $11-$ Me gusta escuchar a las personas que tienen una opinión diferente a la mía- mostró una carga inferior a .40, y el ítem 4 -Tengo curiosidad por las cosas que me rodean y por el mundo en el que vivo- mostró cargas cruzadas entre dos factores. Estos ítems se eliminaron y se volvió a someter a los ítems restantes a ACP. La nueva solución mostró la existencia de asimismo cuatro factores, con dos ítems con cargas pobres: el ítem 8 - La ciencia puede ayudar a que el mundo sea mejor- y el ítem 1 -Merece la pena gastar dinero en la ciencia-. Tras eliminar nuevamente estos ítems, se encontró una solución robusta definitiva compuesta por 10 ítems distribuidos en los siguientes cuatro factores con $\mathrm{K}>1$ y cargas factoriales por encima del criterio 40. En conjunto, estos ítems explican un $65.432 \%$ de la varianza total -ver Tabla 2-.

Tabla 2. Resultados del análisis de componentes principales

\begin{tabular}{|c|c|c|c|c|c|}
\hline \multirow{2}{*}{ Ítems } & \multicolumn{4}{|c|}{ Factores } & \multirow{2}{*}{$h^{2}$} \\
\hline & 1 & 2 & 3 & 4 & \\
\hline $\begin{array}{l}\text { Cuando sea mayor, quiero estudiar algo que } \\
\text { tenga que ver con la ciencia. }\end{array}$ & .788 & & & & .672 \\
\hline $\begin{array}{l}\text { Me gustaría recibir materiales científicos para } \\
\text { poder hacer experimentos en casa }\end{array}$ & .756 & & & & .597 \\
\hline $\begin{array}{l}\text { Cuando sea mayor, me gustaría trabajar } \\
\text { con personas que realizan descubrimientos } \\
\text { científicos }\end{array}$ & .696 & & & & .649 \\
\hline Me gusta hablar sobre la ciencia fuera de clase & .626 & & & & .551 \\
\hline $\begin{array}{l}\text { Ciencias de la Naturaleza es la asignatura más } \\
\text { interesante }\end{array}$ & & .869 & & & .755 \\
\hline $\begin{array}{l}\text { Me gustaría tener más horas de Ciencias de la } \\
\text { Naturaleza a la semana. }\end{array}$ & & .779 & & & .739 \\
\hline $\begin{array}{l}\text { Prefiero resolver un problema haciendo un } \\
\text { experimento en lugar de recibir una respuesta }\end{array}$ & & & .831 & & .686 \\
\hline $\begin{array}{l}\text { Es mejor descubrir la respuesta mediante un } \\
\text { experimento antes que preguntar al profesor }\end{array}$ & & & .740 & & .589 \\
\hline $\begin{array}{l}\text { Un científico se parece mucho a las demás } \\
\text { personas }\end{array}$ & & & & .843 & .693 \\
\hline $\begin{array}{l}\text { Los científicos son igual de simpáticos que las } \\
\text { demás personas }\end{array}$ & & & & .719 & .613 \\
\hline Valores propios - eigenvalues- & 3.048 & 1.311 & 1.162 & 1.023 & - \\
\hline \% Varianza & 30.475 & 13.108 & 11.615 & 10.234 & 65.432 \\
\hline
\end{tabular}

a Los factores representan: 1. Entusiasmo por la ciencia; 2. Actitud hacia las clases de Ciencias de la Naturaleza; 3. Adopción de actitudes científicas; 4. Actitud hacia los científicos

${ }^{\mathrm{b}} h^{2}$ recoge las comunalidades de proporción de la varianza 
Los items que componen estos cuatro factores son:

- Factor 1: Entusiasmo por la ciencia. Este factor está compuesto por cuatro ítems. Los ítems 14 y 7 corresponden al factor original de la escala TOSRA denominado "interés por una carrera en ciencias" y los ítems 6 y 13 al factor original "actitud hacia un ocio en ciencias". Consideramos apropiado nombrar este factor "entusiasmo" debido a que los cuatro ítems están relacionados con el interés hacia asuntos formales o informales relacionados con la ciencia.

- Factor 2: Actitud hacia las clases de Ciencias de la Naturaleza. Se trata de un factor compuesto por 2 ítems que coinciden con el factor original de la escala TOSRA que mide las actitudes hacia la ciencia escolar.

- Factor 3: Adopción de actitudes científicas. Este factor está compuesto por dos ítems que corresponden al factor original de la escala TOSRA que posee el mismo nombre.

- Factor 4: Actitud hacia los científicos. Se trata de un factor compuesto por dos ítems que examinan las actitudes hacia los científicos, y que están en consonancia con el factor original del TOSRA denominado "Normalidad de los científicos".

La fiabilidad del cuestionario es aceptable, tal y como muestra las pruebas de consistencia interna $\alpha$ de Cronbach y correlación ítem-total - ver Tabla 3-. El $\alpha$ de Cronbach total de la escala fue de $\alpha=.725$, con rangos entre .643 y .715, siendo los factores "Actitud hacia las clases de Ciencias de la Naturaleza" y "Actitud hacia los científicos" los que poseen una fiabilidad menor. Las correlaciones ítem-total fueron robustas, con valores por encima del criterio mínimo $r>.30$ para tres de las cuatro escalas. En comparación con los resultados de $\alpha$ de Cronbach de la escala original de Fraser (1981), que fue empleada con estudiantes de Educación Secundaria, los resultados de confiabilidad de esta versión adaptada para Educación Primaria son menores. No obstante, teniendo en cuenta que el $\alpha$ de Cronbach es un prueba muy sensible al número de ítems, estos resultados pueden considerarse aceptables para estudios exploratorios (Nunnally y Bernstein, 1994). En el anexo se recoge el cuestionario definitivo con formato de administración.

Tabla 3. Resultados de confiabilidad

\begin{tabular}{|l|c|c|c|}
\hline \multirow{2}{*}{ Factores } & \multicolumn{3}{|c|}{ Consistencia interna } \\
\cline { 2 - 4 } & $\begin{array}{c}\alpha \text { de Cronbach } \\
\text { TOSRA adaptado }\end{array}$ & $\begin{array}{c}\alpha \text { de Cronbach } \\
\text { TOSRA original }\end{array}$ & $\begin{array}{c}\text { Correlación ítem-total } \\
\text { TOSRA adaptado }\end{array}$ \\
\hline 1. Entusiasmo por la ciencia & .715 & $\begin{array}{l}\text { a } .85-.88 \\
\text { b } .84-.87\end{array}$ & $.590-.729$ \\
\hline $\begin{array}{l}\text { 2. Actitud hacia las clases de } \\
\text { Ciencias de la Naturaleza }\end{array}$ & .663 & $.90-.92$ & .301 \\
\hline $\begin{array}{l}\text { 3. Adopción de actitudes } \\
\text { científicas }\end{array}$ & .715 & $.62-.68$ & .570 \\
\hline 4. Actitud hacia los científicos & .643 & $.63-.73$ & .298 \\
\hline
\end{tabular}

a Los valores pertenecen al constructo original denominado "Interés por una carrera en ciencias"

${ }^{b}$ Los valores pertenecen al constructo original denominado "Actitud hacia un ocio en ciencias"

P1. ¿Cuál es la actitud hacia la ciencia de los estudiantes de Educación Primaria interesados en actividades extracurriculares?

Teniendo en cuenta una puntuación mínima de 1 -actitud negativa- y una puntuación máxima de 4 -actitud positiva-, los estudiantes de Educación Primaria de esta muestra 
poseen actitudes en general muy positivas, especialmente en relación con la adopción de actitudes científicas y con la actitud hacia los científicos. El constructo que ha registrado una valoración más negativa por parte de los estudiantes es el referido a las clases de Ciencias de la Naturaleza, seguido por el entusiasmo por la ciencia - ver Figura 1-.

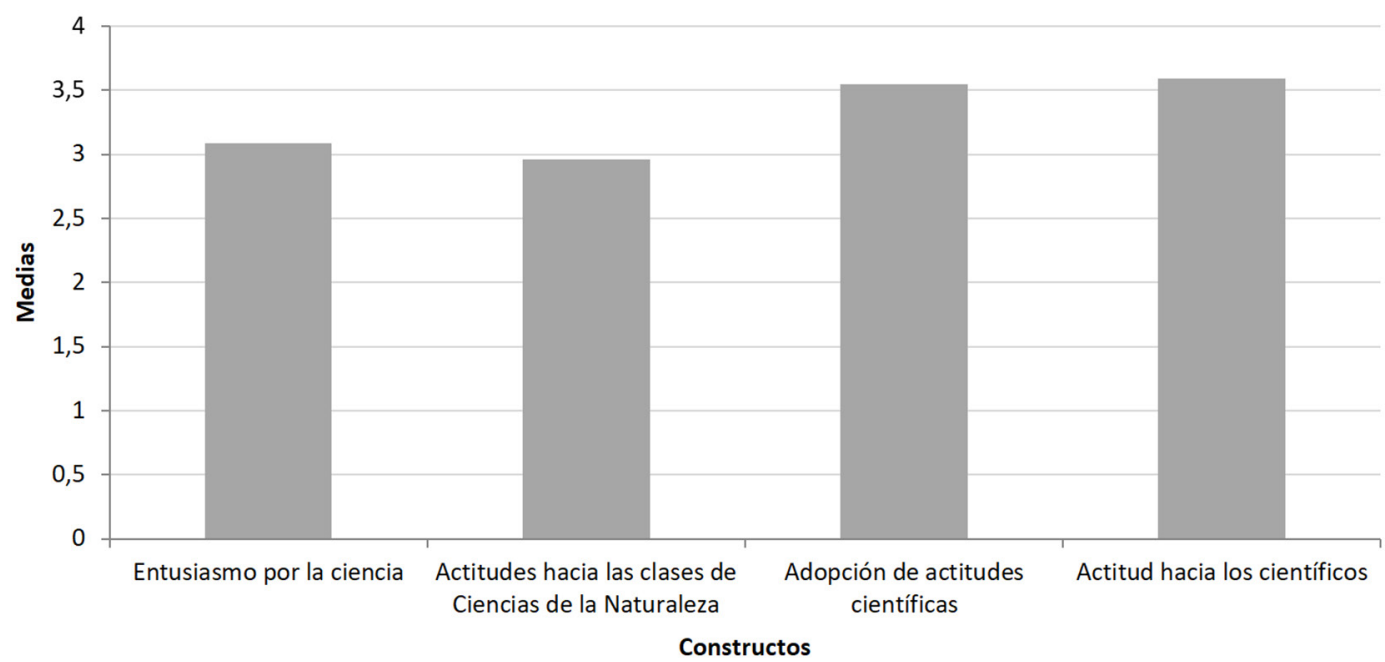

Figura 1. Puntuación media para cada constructo

\section{P2. ¿Difieren las actitudes hacia la ciencia según el género de los estudiantes?}

Tanto las niñas como los niños mostraron actitudes altas equiparables en relación con la dimensión de adopción de actitudes científicas. Sin embargo, las niñas puntuaron un poco más bajo que los niños en los constructos "Entusiasmo por la ciencia" y "Actitud hacia las clases de Ciencias de la Naturaleza" y puntuaron más alto en comparación con los niños en el constructo "Actitud hacia los científicos". El test $t$ de Student para muestras independientes reveló que la única diferencia en alcanzar significancia estadística según la variable género fue la del constructo "Actitudes hacia los científicos". Así, las niñas mostraron unas actitudes significativamente más positivas hacia los científicos que los niños, $t(960)$ $=2.49, p=.01-$ ver Tabla $4-$. Las diferencias registradas en los otros tres constructos no alcanzaron significancia estadística, empleando la corrección de Bonferroni.

Tabla 4. Resultados de la prueba $t$ de Student para la variable género

\begin{tabular}{|c|c|c|c|c|c|}
\hline \multirow{2}{*}{ Factores de la escala TOSRA } & \multirow{2}{*}{ Género } & \multirow{2}{*}{$M$} & \multirow{2}{*}{$D E$} & \multicolumn{2}{|c|}{$t$ de Student } \\
\hline & & & & $t$ & $p$ \\
\hline \multirow{2}{*}{ Entusiasmo por la ciencia } & Niña & 3.05 & .67 & \multirow{2}{*}{-1.78} & \multirow{2}{*}{.076} \\
\hline & Niño & 3.13 & .66 & & \\
\hline \multirow{2}{*}{$\begin{array}{l}\text { Actitud hacia las clases de Ciencias de la } \\
\text { Naturaleza }\end{array}$} & Niña & 2.91 & .85 & \multirow{2}{*}{-1.95} & \multirow{2}{*}{.052} \\
\hline & Niño & 3.02 & .90 & & \\
\hline \multirow{2}{*}{ Adopción de actitudes científicas } & Niña & 3.55 & .64 & \multirow{2}{*}{.311} & \multirow{2}{*}{.756} \\
\hline & Niño & 3.54 & .69 & & \\
\hline \multirow{2}{*}{ Actitud hacia los científicos } & Niña & 3.63 & .57 & \multirow{2}{*}{2.49} & \multirow{2}{*}{.01} \\
\hline & Niño & 3.53 & .67 & & \\
\hline
\end{tabular}

${ }^{a} M$ : media; $D E$ : desviación estándar

${ }^{\mathrm{b}}$ Significancia estadística empleando el ajuste de Bonferroni $(p=.01)$ 


\section{P3. ¿Difieren las actitudes hacia la ciencia según el nivel escolar?}

En relación con el curso escolar, se identificó un descenso en el entusiasmo por la ciencia y en las actitudes hacia las clases de Ciencias de la Naturaleza a partir del 4ㅇ curso de Educación Primaria. La adopción de actitudes científicas se mantiene positiva a lo largo de toda la etapa y las actitudes hacia los científicos mejoran ligeramente en los cursos superiores. La prueba ANOVA reveló la existencia de diferencias significativas en el constructo "Entusiasmo por la ciencia" $F(3,960)=8.22, p<.001$ y "Actitud hacia las clases de Ciencias de la Naturaleza" $F(3,960)=5.66, p<.001$. La prueba post-hoc de Bonferroni reveló que los estudiantes de 5 y 6으 curso de Educación Primaria poseen un entusiasmo por la ciencia significativamente menor que los estudiantes de 3 ㅇ y 40 curso, y actitudes significativamente más desfavorables hacia las clases de Ciencias de la Naturaleza que los estudiantes de 4을 curso - ver Tabla 5-.

Tabla 5. Resultados de la prueba ANOVA para la variable nivel escolar

\begin{tabular}{|l|c|c|c|c|c|c|c|c|}
\hline \multirow{2}{*}{$\begin{array}{l}\text { Curso } \\
\text { escolar }\end{array}$} & \multicolumn{2}{|c|}{\begin{tabular}{c}
\multicolumn{2}{|c|}{$\begin{array}{c}\text { Entusiasmo por } \\
\text { la ciencia* }\end{array}$} \\
\cline { 2 - 9 }
\end{tabular}} & $\begin{array}{c}\text { Actitud hacia las } \\
\text { clases de Ciencias } \\
\text { de la Naturaleza* }\end{array}$ & \multicolumn{2}{c|}{$\begin{array}{c}\text { Adopción } \\
\text { de actitudes } \\
\text { científicas }\end{array}$} & \multicolumn{2}{c|}{$\begin{array}{c}\text { Actitud hacia los } \\
\text { científicos }\end{array}$} \\
\hline 3으. P. & 3.18 & .63 & 2.98 & .95 & 3.58 & .61 & 3.51 & .69 \\
\hline 4으. E. P. & 3.22 & .69 & 3.15 & .85 & 3.54 & .73 & 3.57 & .64 \\
\hline 5으. E. P. & 2.99 & .62 & 2.81 & .87 & 3.48 & .66 & 3.60 & .58 \\
\hline 60 E. P. & 2.93 & .69 & 2.88 & .80 & 3.59 & .62 & 3.66 & .56 \\
\hline
\end{tabular}

${ }^{a} \mathrm{M}$ : media; DE: desviación estándar

* Constructos en los que se han identificado diferencias significativas según el nivel escolar $(p=.01)$

\section{Discusión}

El presente estudio tuvo por objetivo determinar qué actitudes hacia la ciencia poseen alumnos de Educación Primaria que participaron, de forma voluntaria, en una actividad extracurricular de índole científica. Los resultados generales obtenidos en todas las dimensiones son altos. Es muy difícil comparar estos valores con otros de la literatura, pues los diferentes autores no miden exactamente las mismas dimensiones. Sin embargo, si tenemos en cuenta "valores globales", los obtenidos en esta muestra son superiores a los obtenidos por Vázquez Alonso y Manassero Mas (2008), que han evaluado dimensiones similares a las examinadas en este trabajo y con una muestra de edades también semejantes. Aunque hay que tomar con cautela esta comparación, el resultado parecería indicar que los estudiantes que han participado de esta actividad tienen actitudes más positivas hacia la ciencia que otros estudiantes, lo cual era esperable. Asimismo, cabe destacar que, considerando los estudiantes de 60 de Educación Primaria de esta muestra, sus actitudes hacia las clases de Ciencias de la naturaleza son muy similares a los registrados por Marbá-Tallada y Márquez Bargalló (2010), por lo que parecería que la participación en actividades extracurriculares de ciencias no implica una mejora de las actitudes hacia la ciencia escolar.

Analizando los resultados según la variable género, en este estudio no existen diferencias tan marcadas entre las actitudes de los niños y las niñas, a diferencia de lo obtenido por de Pro Bueno y Pérez Manzano (2014) que indicaron visiones más positivas sobre la ciencia en el caso de los niños. Por otra parte, se observa que las niñas parecen disfrutar algo menos durante las clases de ciencias y que poseen un menor entusiasmo por la ciencia 
que los niños. No obstante, las diferencias en relación con estas dos dimensiones no han sido significativas, por lo que no se puede afirmar que los resultados siguen la tendencia general reportada en la literatura, como los estudios de Vázquez Alonso y Manassero Mas (2008) y Marbá-Tallada y Márquez Bargalló (2010) en el que los niños manifestaron disfrutar más durante las clases de ciencias y mostraron un mayor interés por carreras científicas. Por otro lado, las niñas de esta muestra son quienes poseen mejores actitudes hacia los científicos en comparación con los niños, resultado que difiere del estudio de Pérez Manzano y de Pro Bueno (2018) en el que no han obtenido diferencias entre las visiones de los niños y de las niñas con respecto a los científicos.

En comparación con estudios internacionales, la diferencia de las actitudes entre los niños y las niñas no ha sido tan marcada y estos resultados están en consonancia con Babaoğlan y Arıkan (2017), Khishfe y Boujaoude (2016) y Said et al., (2016) que tampoco han encontrado una influencia significativa del género en la formación de actitudes hacia la ciencia. Es posible que estos resultados sean producto de la muestra utilizada: las niñas que han asistido a estas actividades voluntarias podrían estar tan motivadas por actividades científicas cuánto los niños. No obstante, el menor disfrute de las clases de ciencia y entusiasmo por la ciencia por parte de las niñas parecería alinearse con los resultados de la literatura (Boone, 1997; Caleon y Subramaniam, 2008; Denessen et al., 2015; DeWitt y Archer, 2015; George, 2006; Hacieminoglu, 2016; Jarvis y Pell, 2005), aunque nuestros resultados no son concluyentes.

Con respecto a la variable nivel escolar, aunque se han registrado actitudes positivas en todos los cursos de Educación Primaria analizados, en términos globales las actitudes positivas hacia la ciencia disminuyen a medida que aumenta el curso escolar, especialmente a partir del 4을 curso de Educación Primaria, en relación con las dimensiones relativas a las clases de Ciencias de la Naturaleza y hacia el entusiasmo por la ciencia. Estos resultados coinciden con la tendencia identificada tanto a nivel nacional como internacional (Akpınar et al., 2009; Ali et al., 2013; Denessen et al., 2015; DeWitt y Archer, 2015; George, 2006; Said et al., 2016; Vázquez Alonso y Manassero Mas, 2008).

\section{Conclusiones e Implicaciones}

En este trabajo se han examinado las actitudes hacia la ciencia de los estudiantes que participan en actividades científicas extracurriculares, tras realizar un análisis psicométrico del TOSRA adaptado por Toma y Greca (2018). Los resultados de este estudio indican que el TOSRA adaptado es válido y confiable y puede ser empleado para la identificación de las actitudes de los estudiantes de Educación Primaria hacia la ciencia. Empleado este instrumento, se ha obtenido que los estudiantes de esta muestra que acuden de forma voluntaria a una actividad extracurricular de ciencias muestran actitudes globales muy positivas hacia la ciencia. Sin embargo, y a pesar de estar interesados y de contar con apoyo familiar para asistir a ellas, sus actitudes hacia la ciencia siguen el patrón registrado en la literatura en relación con el descenso del entusiasmo por la ciencia y las actitudes favorable hacia las clases de ciencia a medida que aumenta el curso escolar. En este estudio se ha identificado que esta disminución es pronunciada inclusive a partir del 4을 curso de Educación Primaria.

Estos resultados tienen implicaciones de investigación como didácticas. Por un lado, en futuros estudios conviene estudiar qué factores inciden en el disfrute durante las clases de ciencias para así entender por qué las niñas poseen una actitud más negativa hacia esta asignatura en comparación con los niños. Asimismo, es necesario seguir estudiando los factores que intervienen en el desarrollo del entusiasmo por la ciencia, lo que permitiría entender por qué este factor disminuye a medida que aumenta el nivel escolar. Por otro 
lado, los resultados de este estudio reafirman la necesidad de estudiar las actitudes hacia la ciencia en edades más tempranas. Actualmente existen muchos estudios sobre las actitudes hacia la ciencia de los estudiantes, sin embargo, como ya comentamos, la mayoría de ellos han sido desarrollados para la educación secundaria y terciaria.

Desde el punto de vista didáctico, los resultados de este estudio son relevantes, pues resaltan la necesidad de desarrollar intervenciones educativas enfocadas a prevenir el descenso de actitudes favorables desde edades más tempranas, fenómeno que se viene advirtiendo desde hace una década por Tai et al. (2006), entre otros. Así, parece necesario enfocar las intervenciones desde, al menos, 4ㅇ y 5o curso de Educación Primaria, momento en el que se observa un marcado descenso en las actitudes de los estudiantes hacia la ciencia y que parece ser un punto de inflexión en el desarrollo de actitudes favorables.

\section{Agradecimientos}

Este trabajo ha sido parcialmente financiado por la Federación Española de Ciencia y Tecnología (FECyT) a través de los proyectos FCT-16-10972 y FCT-17-12219 y por la Junta de Castilla y León a través del proyecto BU096G18.

\section{Referencias bibliográficas}

Akpınar, E., Yıldız, E., Tatar, N., y Ergin, Ö. (2009). Students' attitudes toward science and technology: an investigation of gender, grade level, and academic achievement. Procedia Social and Behavioral Sciences, 1(1), 2804-2808. DOI: https://doi. org/10.1016/j.sbspro.2009.01.498

Ali, M. M., Yager, R., Hacieminoglu, E., y Caliskan, I. (2013). Changes in student attitudes regarding science when taught by teachers without experiences with a model professional development program. School Science and Mathematics, 113(3), 109119. DOI: https://doi.org/10.1111/ssm.12008

Babaoğlan, B., y Arıkan, T. (2017). Sixth grade students' attitude toward science course. Turkish Journal of Education, 6(2), 68-78. DOI: https://doi.org/10.19128/turje.294844

Boone, W. J. (1997). Science attitudes of selected middle school students in China: a preliminary investigation of https://doi.org/10.19128/turje.294844similarities and differences as a function of gender. School Science and Mathematics, 97(2), 96-103. DOI: https://doi.org/10.1111/j.1949-8594.1997.tb17349.x

Caleon, I. S., y Subramaniam, R. (2005). The impact of a cryogenics-based enrichment programme on attitude towards science and the learning of science concepts. International Journal of Science Education, 27(6), 679-704. DOI: https://doi. org/10.1080/09500690500038306

Caleon, I. S., y Subramaniam, R. (2008). Attitudes towards science of intellectually gifted and mainstream upper primary students in Singapore. Journal of Research in Science Teaching, 45(8), 940-954. DOI: https://doi.org/10.1002/tea.20250

Denessen, E., Vos, N., Hasselman, F., y Louws, M. (2015).The relationship between primary school teacher and student attitudes towards science and technology. Education Research International, 2015, 1-7. DOI: https://doi.org/10.1155/2015/534690

DeWitt, J., y Archer, L. (2015). Who aspires to a science career? A comparison of survey responses from primary and secondary school students. International Journal of Science Education, 37(13), 2170-2192. DOI: https://doi.org/10.1080/09500693.20 15.1071899 
European Commission. (2015). Science education for Responsible Citizenship [versión PDF]. DOI: https://doi.org/10.2777/12626

Fraser, B. J. (1981). Test of science-related attitudes (TOSRA) handbook. Melbourne, Australia: Australian Council for Educational Research.

George, R. (2006). A cross-domain analysis of change in students' attitudes toward science and attitudes about the utility of science. International Journal of Science Education, 28(6), 571-589. DOI: https://doi.org/10.1080/09500690500338755

Greca, I. M., Diez-Ojeda, M. y García Terceño, E. (en revisión). Evaluación de un proyecto de educación no formal de fomento de actitudes positivas hacia las ciencias y de formación en la enseñanza de las ciencias por indagación.

Guzey, S. S., Moore, T. J., Harwell, M., y Moreno, M. (2016). STEM integration in middle school life science: student learning and attitudes. Journal of Science Education and Technology, 25(4), 550-560. DOI: https://doi.org/10.1007/s10956-016-9612-x

Hacieminoglu, E. (2016). Elementary school students' attitude toward science and related variables. International Journal of Environmental \& Science Education, 11(2), 35-52. DOI: https://doi.org/10.12973/ijese.2016.288a

Huang, NT. N., Chiu, LJ., y Hong, JC. (2016). Relationship among students' problem-solving attitude, perceived value, behavioral attitude, and intention to participate in a science and technology contest. International Journal of Science and Mathematics Education, 14(8), 1419-1435. DOI: https://doi.org/10.1007/s10763-015-9665-y

Jarvis, T., y Pell, A. (2005). Factors influencing elementary school children's attitudes toward science before, during, and after a visit to the UK National Space Centre. Journal of Research in Science Teaching, 42(1), 53-83. DOI: https://doi.org/10.1002/tea.20045

Jones, M. G., Howe, A., y Rua, M. J. (2000). Gender differences in students' experiences, interests, and attitudes toward science and scientists. Science Education, 84(2), 180-192. DOI: https://doi.org/10.1002/(SICI)1098-237X(200003)84:2<180::AIDSCE3>3.0.CO;2-X

Junta de Castilla y León. (2018). Relación de centros educativos que imparten enseñanzas no universitarias en el curso académico actual [Archivo de datos]. Recuperado de: https://datosabiertos.jcyl.es/web/jcyl/set/es/educacion/ centrosdocentes/1284200521439

Kaiser, H. F. (1970). A second-generation little jiffy. Psychometrika, 35(4), 401-415. DOI: https://doi.org/10.1007/BF02291817

Khishfe, R., y Boujaoude, S. (2016). Lebanese students' conceptions of and attitudes towards science and related careers based on their gender and religious affiliations. International Journal of Science and Mathematics Education, 14(Supplement 1), 145-167. DOI: https://doi.org/10.1007/s10763-014-9587-0

Klopfer, L. E. (1971). Evaluation of learning in science. En B. S. Bloom, J. T. Hastings y G. F. Madaus (Eds.), Handbook on Summative and Formative Evaluation of Student Learning (pp. 559-641). Nueva York, NY: McGraw-Hill.

Lloret-Segura, S., Ferreres-Traves, A., Hernández-Baeza, A., y Tomás-Marco, I. (2014). El análisis factorial exploratorio de los ítems: una guía práctica, revisada y actualizada. Anales de Psicología, 30(3), 1151-1169. DOI: https://doi.org/10.6018/ analesps.30.3.199361 
Marbà-Tallada, A., y Márquez Bargalló, C. (2010). ¿Qué opinan los estudiantes de las clases de ciencias? Un estudio transversal de sexto de primaria a cuarto de ESO. Enseñanza de las Ciencias, 28(1), 19-30. Recuperado de: https://ddd.uab.cat/pub/ edlc/02124521v28n1/02124521v28n1p19.pdf

Méndez Martínez, C., y Rondón Sepúlveda, M. A. (2012). Introducción al análisis factorial exploratorio. Revista Colombiana de Psiquiatría, 41(1), 197-207. DOI: https://doi. org/10.1016/S0034-7450(14)60077-9

Mıhladız, G., Duran, M., y Doğan, A. (2011). Examining primary school students' attitudes towards science in terms of gender, class level and income level. Procedia Social and Behavioral Sciences, 15, 2582-2588. DOI: https://doi.org/10.1016/j. sbspro.2011.04.150

Molina, M., Carriazo, J., y Casas, J. (2013). Estudio transversal de las actitudes hacia la ciencia en estudiantes de grados quinto a undécimo. Adaptación y aplicación de un instrumento para valorar actitudes. Tecné, Episteme y Didaxis, 2013(33), 103-122. DOI: https://doi.org/10.17227/01213814.33ted103.122

Newell, A. D., Zientek, L. R., Tharp, B. Z., Vogt, G. L., y Moreno, N. P. (2015). Students' attitudes toward science as predictors of gains on student content knowledge: Benefits of an after-school program. School Science and Mathematics, 115(5), 216225. DOI: https://doi.org/10.1111/ssm.12125

Nortes Martínez-Artero, R., y de Pro Bueno, A. (2010). Actitudes hacia las ciencias de los alumnos de Educación Primaria de la Región de Murcia. II Jornadas de los Máster en Investigación e Innovación en Educación Infantil y Educación Primaria, 441-464. Recuperado de: https://www.um.es/c/document_library/get_file?uuid=d3208f2720f1-4da5-af3a-975da73e1853\&groupld=299436

Nunnally, J. C., y Bernstein, I. H. (1994). Psychometric theory. Nueva York, Estados Unidos: McGraw-Hill.

Ortiz-Revilla, J., Greca, I. M., y Adúriz-Bravo, A. (2017). Presencia y caracterización de las competencias cientifica y matemática en estudios sobre Educación Primaria. Ponencia presentada en el I Congreso Nacional en Enseñanza de las Ciencias de la Naturaleza y la Matemática y II Congreso Regional en Enseñanza de las Ciencias de la Naturaleza, Tandil, Argentina.

Ortiz-Revilla, J., Greca, I. M., y Adúriz-Bravo, A. (2018). La Educación STEAM y el desarrollo competencial en la Educación Primaria. En I. M. Greca y J. Á. Meneses Villagrá (Eds.), Proyectos STEAM para la Educación Primaria. Fundamentos y aplicaciones prácticas (pp. 41-54). Madrid, España: Dextra.

Osborne, J., y Dillon, J. (2008). Science education in Europe: Critical reflections. Recuperado de: http://www.nuffieldfoundation.org/sites/default/files/files/Sci_Ed_in_Europe_ Report_Final(1).pdf

Pérez-Franco, D., de Pro-Bueno, A. J., y Pérez-Manzano, A. (2018). Actitudes ambientales al final de la ESO. Un estudio diagnóstico con alumnos de Secundaria de la Región de Murcia. Revista Eureka sobre Enseñanza y Divulgación de las Ciencias, 15(3), 1-17. DOI: https://doi.org/10.25267/Rev_Eureka_ensen_divulg_cienc.2018.v15.i3.350

Pérez Manzano, A., y de Pro Bueno, A. (2018). Algunos datos sobre la visión de los niños y de las niñas sobre las ciencias y del trabajo científico. iQual. Revista de Género e Igualdad, 1, 18-31. DOI: https://doi:10.6018/iQual.306091 
de Pro Bueno, A., y Pérez Manzano, A. (2014). Actitudes de los alumnos de Primaria y Secundaria ante la visión dicotómica de la Ciencia. Enseñanza de las Ciencias, 32(3), 111-132. DOI: https://doi.org/10.5565/rev/ensciencias.1015

Said, Z., Summers, R., Abd-El-Khalick, F., y Wang, S. (2016). Attitudes toward science among grades 3 through 12 Arab students in Qatar: Findings from a cross-sectional national study. International Journal of Science Education, 38(4), 621-643. DOI: https://doi. org/10.1080/09500693.2016.1156184

Tabachnick, B. G., y Fidell, L. S. (2007). Using multivariate statistics. Boston, MA: Pearson.

Tai, R. H., Liu, C. Q., Maltese, A. V., y Fan, X. (2006). Planning early for careers in Science. Science, 312(5777), 1143-1144. DOI: https://doi.org/10.1126/science.1128690

Toma, R. B., y Greca, I. M. (2018). The effect of integrative STEM instruction on elementary students' attitudes toward science. Eurasia Journal of Mathematics, Science and Technology Education, 14(4), 1383-1395. DOI: https://doi.org/10.29333/ ejmste/83676

Toma, R. B., Greca, I. M., y Orozco Gómez, M. L. (2019). Attitudes towards science and views of nature of science among elementary school students in terms of gender, cultural background and grade level variables. Research in Science \& Technological Education. DOI: https://doi.org/10.1080/02635143.2018.1561433

Vázquez Alonso, Á., Acevedo Díaz, J. A., Manassero Mas, M. A., y Acevedo Romero, P. (2006). Actitudes del alumnado sobre ciencia, tecnología y sociedad, evaluadas con un modelo de respuesta múltiple. Revista Electrónica de Investigación Educativa, 8(2), 1-37. Recuperado de: https://redie.uabc.mx/redie/article/view/145/250

Vázquez Alonso, Á., y Manassero Mas, M. A. (1997). Una evaluación de las actitudes relacionadas con la ciencia. Enseñanza de las Ciencias, 15(2), 199-213. Recuperado de: https://ddd.uab.cat/pub/edlc/02124521v15n2/ 02124521v15n2p199.pdf

Vázquez Alonso, Á., y Manassero Mas, M. A. (2008). El declive de las actitudes hacia la ciencia de los estudiantes: un indicador inquietante para la educación científica. Revista Eureka sobre Enseñanza y Divulgación de las Ciencias, 5(3), 274-292. Recuperado de: http://revistas.uca.es/index.php/eureka/article/view/3740 


\section{Anexo: escala TOSRA adaptada}

Rodea el número que represente tu grado de acuerdo o desacuerdo con las siguientes frases, siendo:

1. Totalmente en desacuerdo

2. En desacuerdo

3. De acuerdo

4. Totalmente de acuerdo

\begin{tabular}{lllll}
\hline 1. Me gusta hablar sobre la ciencia fuera de clase & 1 & 2 & 3 & 4 \\
\hline 2. Ciencias de la Naturaleza es la asignatura más interesante & 1 & 2 & 3 & 4 \\
\hline $\begin{array}{l}\text { 3. Prefiero resolver un problema haciendo un experimento en lugar de } \\
\text { recibir una respuesta }\end{array}$ & 1 & 2 & 3 & 4 \\
\hline $\begin{array}{l}\text { 4. Un científico se parece mucho a las demás personas } \\
\text { 5. Cuando sea mayor, quiero estudiar algo que tenga que ver con la ciencia. }\end{array}$ & 1 & 2 & 3 & 4 \\
\hline $\begin{array}{l}\text { 6. Me gustaría tener más horas de Ciencias de la Naturaleza a la semana. } \\
\text { 7. Es mejor descubrir la respuesta mediante un experimento antes que }\end{array}$ & 1 & 2 & 3 & 4 \\
\hline preguntar al profesor & 1 & 2 & 3 & 4 \\
\hline $\begin{array}{l}\text { 8. Los científicos son igual de simpáticos que las demás personas } \\
\text { 9. Me gustaría recibir materiales científicos para poder hacer experimentos } \\
\text { en casa }\end{array}$ & 1 & 2 & 3 & 4 \\
\hline $\begin{array}{l}\text { 10. Cuando sea mayor, me gustaría trabajar con personas que realizan } \\
\text { descubrimientos científicos }\end{array}$ & 1 & 2 & 3 & 4 \\
\hline
\end{tabular}

Factor 1: Entusiasmo por la ciencia $=\frac{\text { ítem 1+ítem 5+ítem 9+ítem } 10}{4}$

Factor 2: Actitud hacia las clases de Ciencias de la Naturaleza $=\frac{\text { ítem } 2+\text { ítem } 6}{2}$

Factor 3: Adopción de actitudes científicas $=\frac{\text { ítem } 3+\text { ítem } 7}{2}$

Factor 4: Actitud hacia los científicos $=\frac{\text { ítem } 4+\text { ítem } 8}{2}$ 
